\title{
Evaluación de análisis de endemicidad y PAE para identificar trazos generalizados, a partir de las serpientes del estado de Hidalgo, México
}

\author{
Gustavo Montiel-Canales ${ }^{1}$, Irene Goyenechea Mayer-Goyenechea ${ }^{1 *}$, Leonardo-Fernández- \\ Badillo $^{1,2}$ \& Jesús M. Castillo Cerón ${ }^{3}$ \\ 1. Laboratorio de Sistemática Molecular, Centro de Investigaciones Biológicas (CIB), Universidad Autónoma del Estado \\ de Hidalgo, Km 4.5 Carretera Pachuca-Tulancingo s/n Col. Carboneras, C.P. 42181, Mineral de la Reforma, Hidalgo, \\ México; gmoncana@gmail.com, ireneg28@gmail.com* \\ 2. Predio intensivo de manejo de vida silvestre X-plora Reptilia, Reyna de la Esperanza \#107, Col. Paseo de las Reinas \\ $1^{a}$ sección. C. P. 42184, Mineral de la Reforma, Hidalgo, México; cyrtopsis@hotmail.com \\ 3. Museo de Paleontología, Centro de Investigaciones Biológicas (CIB), Universidad Autónoma del Estado de Hidalgo, \\ Km 4.5 Carretera Pachuca-Tulancingo s/n Col. Carboneras, C.P. 42181, Mineral de la Reforma, Hidalgo, México; \\ castjmc@gmail.com \\ * Correspondence
}

Recibido 16-IX-2015. Corregido 05-IV-2016. Aceptado 06-V-2016.

\begin{abstract}
Evaluation of PAE and endemicity analysis for identifying generalized tracks using snakes in Hidalgo, Mexico. One of the most important concepts in Panbiogeography is the generalized track, which represents an ancestral biota fragmented by geological events that can be recovered through several methods, including Parsimony analysis of endemicity (PAE) and endemicity analysis (EA). PAE has been frequently used to identify generalized tracks, while EA is primarily designed to find areas of endemicity, but has been recently proposed for identifying generalized tracks as well. In this study we evaluated these methods to find generalized tracks using the distribution of the 84 snake species of Hidalgo. PAE found one generalized track from three individual tracks (Agkistrodon taylori, Crotalus totonacus and Pliocercus elapoides), supported by $89 \%$ of Bootstrap, and EA identified two generalized tracks, with endemicity index values of 2.71-2.96 and 2.84-3.09, respectively. Those areas were transformed to generalized tracks. The first generalized track was retrieved from three individual tracks (Micrurus bernadi, Rhadinaea marcellae and $R$. quinquelineata), and the second was recovered from two individual tracks (Geophis mutitorques and Thamnophis sumichrasti). These generalized tracks can be considered a unique distribution pattern, because they resembled each other and agreed in shape. When comparing both methods, we noted that both are useful for identifying generalized tracks, and although they can be used independently, we suggest their complementary use. Nevertheless, to obtain accurate results, it is useful to consider theoretical bases of both methods, along with an appropriate choice of the size of the area. Results using small-grid size in EA are ideal for searching biogeographical patterns within geopolitical limits. Furthermore, they can be used for conservation proposals at state level where endemic species become irreplaceable, and where losing them would imply the extinction of unique lineages. Rev. Biol. Trop. 64 (4): 1611-1624. Epub 2016 December 01.
\end{abstract}

Key words: Mexico, panbiogeography, biotic components, parsimony analysis of endemicity, endemicity analysis.

Los análisis de la distribución geográfica de los seres vivos y el reconocimiento de los componentes bióticos resultan claves para comprender la evolución en espacio-tiempo debido a una historia evolutiva común (Craw, 1988; Morrone, 2004). La panbiogeografía (Craw, 1988) es un enfoque histórico que surgió como reacción al dispersalismo de Darwin (1859) y Wallace (1876). De acuerdo con Croizat (1958, 1964), este método asume que las biotas evolucionan de acuerdo a los cambios en las barreras geográficas, lo que se resume en la idea general de que "Tierra y biota evolucionan juntas" (Morrone, 2000), aunque debe considerarse 
también a la dispersión como un componente para comprender la distribución y evolución de las biotas (Morrone, 2015).

Uno de los conceptos centrales en la Panbiogeografía es el trazo generalizado, el cual representa una biota ancestral que se ha fragmentado por eventos geológicos (Morrone, 2004; 2015). A lo largo del tiempo se han utilizado diferentes métodos para identificar dichos trazos generalizados (Fernández-Badi1lo, Goyenechea, \& Escalante, 2014; Morrone, 2001. El primero de ellos fue el procedimiento manual de Croizat (1958, 1964). Posteriormente, surgieron otros como el método de matrices de conectividad e incidencia de Page (1987), el método de compatibilidad de trazos (Craw, 1988) y el análisis de simplicidad de endemismos o PAE (Morrone \& Escalante, 2009), del cual se hizo una reinterpretación llamada PAE-PCE con eliminación progresiva de caracteres (Luna-Vega, Alcántara, Morrone, \& Espinosa, 2000; García-Barros et al., 2002). Existen algunas herramientas computarizadas para aplicarlos, como la extensión Trazos 2004® (Rojas-Parra, 2007) del programa ArcView GIS 3.2 (ESRI, 1998), el método mixto propuesto por Liria (2008), que utiliza sistemas de información y análisis espacial, Croizat (Calvanti, 2009) y MartiTracks (EcheverríaLondoño \& Miranda-Esquivel, 2011).

El PAE ha sido ampliamente utilizado y es considerado como un buen método para identificar los trazos generalizados (Morrone, 2014, 2015), ya que permite delimitar áreas de endemismo, las cuales son análogas a los trazos generalizados, porque en ambos conceptos es necesario detectar la congruencia distribucional en dos o más táxones (García-Marmolejo, Escalante, \& Morrone, 2008). El PAE permite construir cladogramas de áreas mediante análisis cladísticos de matrices de datos de presencia y ausencia de táxones, donde los trazos individuales que evidencian el agrupamiento de áreas son considerados como trazos generalizados (Craw, Grehan, \& Heads, 1999; Luna-Vega et al., 2000; Crisci, Katinas, \& Posadas, 2000; Escalante \& Morrone, 2003; Morrone, 2004). Sin embargo, el PAE ha recibido una variedad de críticas generales a sus diversas aplicaciones, basadas en el mal entendimiento de sus bases teóricas (Morrone, 2014).

Fernández-Badillo et al. (2014) propusieron el uso del análisis de endemicidad o AE (Szumik, Cuezzo, Goloboff, \& Chalup, 2002; Szumik \& Goloboff, 2004) para la identificación de trazos generalizados. El AE aplica un algoritmo de optimización basado en el concepto de áreas de endemismo, que se consideran áreas geográficas delimitadas por la congruencia en los intervalos de distribución de al menos dos táxones (Szumik, Casagranda, \& Roig-Juñet, 2006). Este algoritmo permite evaluar cuántos y qué tan endémicos son los táxones de un área determinada, de manera que aquellas áreas caracterizadas por al menos dos táxones e índices de endemicidad, serán seleccionadas como áreas de endemismo (Szumik et al., 2002; Szumik \& Goloboff, 2004; Szumik et al., 2006). Éste análisis se implementa en los programas NDM/VNDM (Goloboff, 2014). El $\mathrm{AE}$ se desarrolló en un principio para la identificación de áreas de endemismo, pero si se considera que éstas son equivalentes a los trazos generalizados (García-Marmolejo et al., 2008), entonces el AE también puede ser utilizado como un método para la detección de trazos generalizados (Fernández-Badillo et al., 2014).

La propuesta de Fernández-Badillo et al. (2014) para detectar trazos generalizados mediante el AE sugiere cambiar los parámetros de búsqueda para las áreas de endemismo al asignar un valor de cero para el caso de los registros inferidos y un valor de diez para los registros asumidos, así como la modificación del valor mínimo de endemismo para el área a 1.5. A las áreas de endemismo resultantes se les debe aplicar la opción de "áreas consenso" (para mayores detalles del método ver Fernández-Badillo et al., 2014).

Hasta el momento no existen comparaciones directas de la aplicación del AE y el PAE para obtener los trazos generalizados. Sin embargo, existen algunos trabajos en México como los de Escalante, Szumik y Morrone (2007, 2009) y Fernández-Badillo (2013), en los que se utilizó el AE como una alternativa 
al PAE para la búsqueda de áreas de endemismo y se obtuvieron resultados favorables, ya que se identificaron áreas sustentadas por un mayor número de táxones. Esto sugiere que el AE presenta ventajas sobre el PAE.

El estado de Hidalgo es uno de los estados más pequeños de México; cuenta con una superficie territorial de $20905 \mathrm{~km}^{2}$, lo que representa el $1.1 \%$ del total del país (INEGI, 2009). Sin embargo, su ubicación geográfica en el centro del país aunado a su compleja historia geológica, ha propiciado una gran variedad de tipos de vegetación y climas que se reflejan en una elevada diversidad de grupos biológicos. Las serpientes son uno de los grupos que se encuentra bien representado en el estado, con 84 especies descritas (RamírezBautista et al., 2014), de las cuales el $50 \%$ son endémicas a México.

Estos elementos hacen del estado de Hidalgo una área de interés científico, en donde se solapan faunas neárticas y neotropicales (Delgado \& Márquez, 2006). Además, al estar inmerso en diferentes provincias biogeográficas (Ramírez-Bautista, Hernández-Salinas, García-Chávez, Leyte-Manrique, \& CansecoMárquez, 2009) se incrementa el interés de realizar estudios biogeográficos que consideren a la diversidad de especies distribuidas en los límites geopolíticos del estado. Se sabe que las entidades geopolíticas en la mayoría de los casos no representan áreas naturales, pero pueden ser utilizadas con el fin de generar información para realizar estrategias de conservación a nivel estatal (Cué-Bär, Villaseñor, Morrone, \& Ibarra-Manríquez, 2006), ya que son las unidades básicas utilizadas para la delimitación y creación de Áreas Naturales Protegidas (Almazán-Núñez, López-De Aquino, Ríos-Muñoz, \& Navarro-Sigüenza, 2013). En ese sentido, resulta válido buscar patrones de distribución dentro de los límites políticos estatales.

El objetivo de este trabajo fue evaluar el análisis de simplicidad de endemismos (Morrone, 2015) y el análisis de endemicidad (Szumik \& Goloboff, 2004) a través del enfoque propuesto por Fernández-Badillo et al. (2014) para identificar trazos generalizados a partir de las serpientes distribuidas en el estado de Hidalgo, con la finalidad de identificar semejanzas y diferencias en la forma en la que se componen los patrones de distribución.

\section{MATERIALES Y MÉTODOS}

El estudio estuvo enfocado en las serpientes distribuidas en el estado de Hidalgo, pero se tomaron en cuenta todos los registros de su distribución en México, con la finalidad de encontrar patrones biogeográficos.

Datos: La lista de las especies de serpientes de Hidalgo se tomó de Ramírez-Bautista et al. (2014) quienes registraron un total de 84 especies pertenecientes a ocho familias y 43 géneros (Cuadro 1). El arreglo taxonómico y nomenclatural de las serpientes fue realizado a nivel de especie de acuerdo con Wilson, MataSilva y Johnson (2013) y Ramírez-Bautista et al. (2014). Se obtuvieron 7357 registros, con un promedio de 87 datos de distribución geográfica por especie, los cuales fueron obtenidos a través de la revisión de la literatura y a partir de la consulta de bases de datos de los proyectos de CONABIO y las bases de datos en línea: Global Biodiversity Information Facility (GBIF, 2014), VertNet (VertNet, 2014), Reptile data base (Uetz \& Hošek, 2014), así como la base de datos del proyecto FOMIX CONACyT 191908 Diversidad biológica del estado de Hidalgo (tercera etapa) revisadas durante el 2014. Además, se corroboró que los registros coincidieran con la distribución conocida de cada especie, para lo cual se compararon con mapas de distribución a partir de la revisión de la literatura y mapas en línea (CONABIO, 2004; IUCN, 2014; ZenScientist, 2014), con la finalidad de eliminar las áreas donde no se hubiera registrado su presencia de manera fidedigna.

Identificación de trazos generalizados: A partir de los registros de distribución, se realizaron los trazos individuales de las 84 serpientes (material complementario). Los trazos se dibujaron a través de la extensión 
CUADRO 1

Especies de serpientes distribuidas en Hidalgo

TABLE 1

Snakes distributed in Hidalgo

1. Boa imperator Familia Boidae

\section{Familia Colubridae}

2. Coluber constrictor

3. Conopsis biserialis

4. C. lineata

5. C. nasus

6. Drymarchon melanurus

7. Drymobius chloroticus

8. D. margaritiferus

9. Ficimia hardyi

10. F. olivacea

11. F. streckeri

12. Lampropeltis mexicana

13. L. polyzona

14. L. ruthveni

15. Leptophis diplotropis

16. L. mexicanus

17. Masticophis schotti

18. Mastigodryas melanolomus

19. Oxybelis aeneus

20. Pantherophis emoryi

21. Pseudelaphe flavirufa

22. Pituophis deppei

23. Salvadora bairdi

24. S. grahamiae

25. Senticolis triaspis

26. Spilotes pullatus

27. Tantilla bocourti

28. T. rubra

29. Trimorphodon tau

30. Agkistrodon taylori

31. Atropoides nummifer

32. Bothrops asper

33. Crotalus aquilus

34. C. atrox

35. C. intermedius

36. C. molossus

37. C. polystictus

38. C. ravus

39. C. scutulatus

40. C. totonacus

41. C. triseriatus

42. Ophryacus undulatus

\section{Familia Dipsadidae}

43. Adelphicos quadrivirgatum

44. Amastridium sapperi

45. Coniophanes fissidens

46. C. imperialis

47. C. piceivittis

48. Chersodromus rubriventris

49. Diadophis punctatus

50. Geophis latifrontalis

51. G. mutitorques

52. G. semidoliatus

53. G. turbidus

54. Hypsiglena jani

55. Imantodes cenchoa

56. I. gemmistratus

57. Leptodeira maculata

58. L. septentrionalis

59. Ninia diademata

60. Pliocercus elapoides

61. Rhadinaea gaigeae

62. $R$. marcellae

63. $R$. quinquelineata

64. Tropidodipsas sartorii

Familia Elapidae

65. Micrurus bernadi

66. M. diastema

67. M. tener

Familia Leptotyphlopidae

68. Epicta goudotii

69. Rena dulcis

70. Rena myopica

71. Nerodia rhombifer

72. Storeria dekayi

73. S. hidalgoensis

74. S. storerioides

75. Thamnophis cyrtopsis

76. T. eques

77. T. marcianus

78. T. melanogaster

79. T. proximus

80. T. pulchrilatus

81. T. scalaris

82. T. scaliger

83. T. sumichrasti

Familia Typhlopidae

84. Indotyphlops braminus 
Trazos2004 (Rojas-Parra, 2007) en el programa ArcView (ESRI, 1998).

Para identificar los trazos generalizados con el PAE, se construyó una matriz de las 14 provincias biogeográficas (Morrone, 2001; Morrone, 2005) por los 84 trazos individuales, donde las columnas representaron los trazos individuales (caracteres), y las filas las áreas comparadas (provincias biogeográficas; material complementario). Se utilizaron las provincias debido a que existen pocos datos puntuales de la distribución de las serpientes, y porque se ha visto que el PAE se desempeña mejor cuando se usan unidades naturales (provincias biogeográficas) en lugar de cuadrículas (Morrone \& Escalante, 2002). Además, el uso de cuadrículas podría haber oscurecido los patrones encontrados, debido a que la naturaleza de los datos puede generar un sesgo en las distribuciones cuando se cuenta con pocos datos puntuales (Rojas-Parra, Poveda-Matallana, Prieto-Cruz, Rudas-Lleras, \& Luis-Martínez, 2003). Se añadió un área hipotética externa con valores de 0 para enraizar los árboles (Morrone, 1994).

La matriz se analizó con un algoritmo de simplicidad en el programa TNT (Goloboff, Farris, \& Nixon, 2008). La obtención de los cladogramas se realizó a partir de una búsqueda heurística con 1000 réplicas de adición de secuencias al azar, intercambio de ramas por TBR (Tree bisection and reconnection) y se retuvieron 10 árboles por réplica. Una vez obtenidos los cladogramas, se construyó el cladograma de consenso estricto (Morrone, 2003). La confiabilidad de los resultados se determinó mediante la aplicación de una prueba estadística de robustez "Bootstrap" con 10000 réplicas (Morrone, 2003). Se identificaron los clados que estuvieron sustentados por al menos dos trazos individuales. Con base en las especies coincidentes se verificó que los trazos individuales de las especies que definen el clado principal formaran un patrón de distribución congruente. Por último, el trazo generalizado obtenido se representó en un mapa.

Para identificar los trazos generalizados con el AE se utilizaron los mismos datos de distribución con los que se construyeron los trazos pero, debido a que el método precisa forzosamente de la utilización de cuadrículas, se utilizó una cuadrícula de 0.5 × 0.5 , es decir una escala más fina que las provincias (material complementario), y se analizó con el software NDM/VNDM (Goloboff, 2014). La búsqueda de áreas de endemismo se realizó con los parámetros sugeridos por FernándezBadillo et al. (2014) para la detección de trazos generalizados: registros inferidos $=0$; registros asumidos $=10$; valor mínimo de endemismo $=1.5$; mantener áreas superpuestas $=40 \%$; áreas consenso $=60 \%$; repeticiones $=1000$. Se usó el consenso del $60 \%$ puesto que éste es un consenso semi-estrecho que permite identificar patrones biogeográficos definidos a partir de la regla del consenso estrecho y que permite asegurar consistencia entre las áreas encontradas (Aagesen, Szumik, \& Goloboff, 2013). Además, de acuerdo con los autores este consenso es una opción cuando se usan escalas pequeñas a nivel regional. Se visualizaron en mapas los trazos individuales que conforman las áreas consenso para establecer que los trazos individuales formaran verdaderamente un patrón congruente de distribución de acuerdo al concepto del trazo generalizado.

\section{RESULTADOS}

A través del PAE se obtuvieron tres cladogramas igualmente parsimoniosos con 200 pasos de longitud, el árbol de consenso estricto contó con una longitud de 205 pasos. Se observaron dos grandes clados A y B (Fig. 1). El clado A involucra a todas las provincias de México con las especies de serpientes que presentaron una distribución más amplia. El clado B se encuentra conformado por las provincias que convergen en el estado de Hidalgo y está apoyado por cuatro sinapomorfías (Agkistrodon taylori, Crotalus totonacus, Micrurus bernadi y Pliocercus elapoides). La prueba de Bootstrap tiene un soporte del $89 \%$. El Golfo de México y la Sierra Madre Oriental son las provincias más cercanamente relacionadas; éstas a su vez forman el grupo hermano del Altiplano 


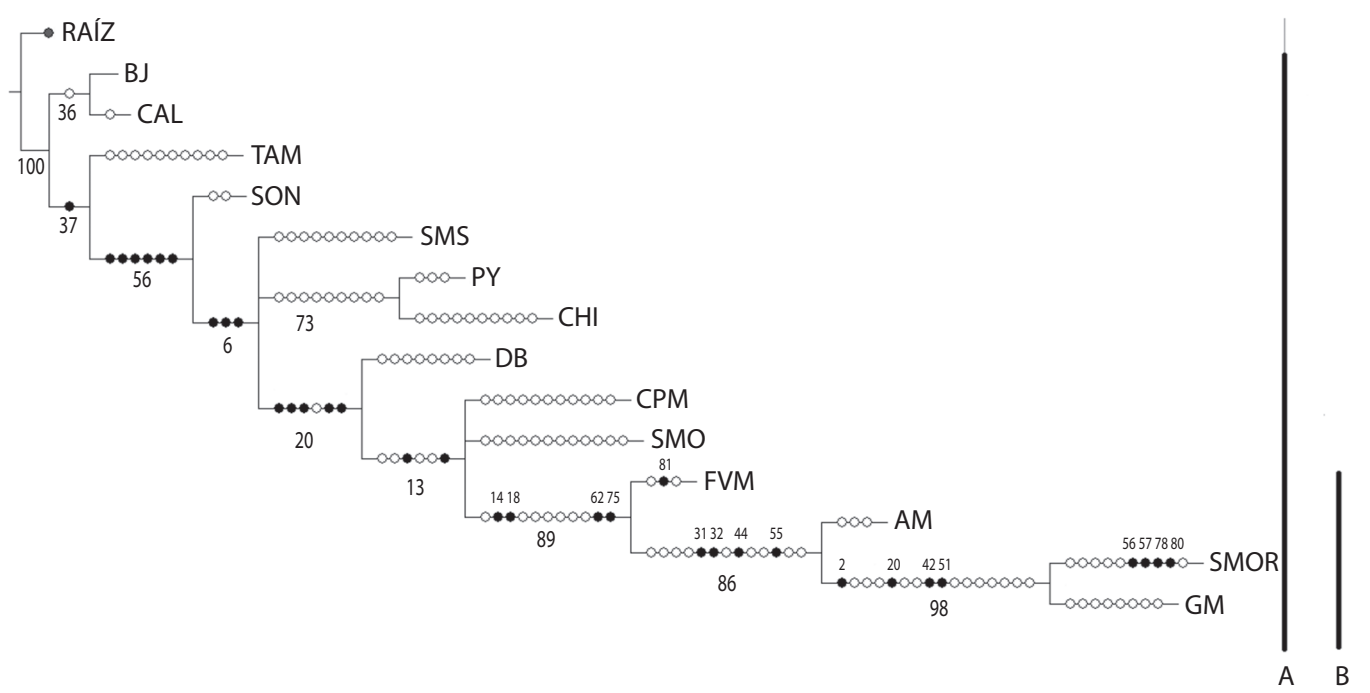

Fig. 1. Cladograma de consenso estricto con las relaciones biogeográficas de las serpientes distribuidas en Hidalgo $\mathbf{B J}=$ Baja California, $\mathbf{C A L}=$ California, $\mathbf{T A M}=$ Tamaulipas, $\mathbf{S O N}=$ Sonora, $\mathbf{S M S}=$ Sierra Madre del Sur, $\mathbf{P Y}=$ Península de Yucatán, $\mathbf{C H I}=$ Chiapas, $\mathbf{D B}=$ Depresión del Balsas, $\mathbf{C P M}=$ Costa del Pacifico Mexicano, $\mathbf{S M O}=$ Sierra Madre Occidental, $\mathbf{F V M}=$ Faja Volcánica, Transmexicana, $\mathbf{A M}=$ Altiplano Mexicano, $\mathbf{S M O R}=$ Sierra Madre Oriental y $\mathbf{G M}=$ Golfo de México. El clado A incluye las 14 provincias, el clado B muestra las relaciones de las provincias en Hidalgo. En negro se muestran las sinapomorfías que forman trazos generalizados: $\mathbf{1 4}=$ Crotalus aquilus, $\mathbf{1 8}=$ Crotalus ravus, $\mathbf{6} \mathbf{1}=$ Storeira hidalgoensis, $\mathbf{7 5}=$ Thamnophis sumichrasti, 31= Geophis mutitorques, 32=Geophis semidoliatus, $\mathbf{4 4}=$ Micrurus tener, 55=Rhadinea gaigeae, $\mathbf{2}=$ Agkistrodon taylori, $\mathbf{2 0}=$ Crotalus totonacus, $\mathbf{4 2}=$ Micrurus bernadi, $\mathbf{5 1}=$ Pliocercus elapoides, $\mathbf{5 6}=$ Rhadinaea marcellae, $\mathbf{5 7}=$ Rhadinaea quinquelineata, $\mathbf{7 8}=$ Chersodromus rubriventris $\mathbf{8 0}=$ Geophis turbidus $\mathbf{8 1}=$ Lampropeltis ruthveni y en blanco se incluyen las homoplasias. Los valores de bootstrap se muestran debajo de los nodos.

Fig. 1. Strict consensus cladogram with biogeographical relationships within snakes' distribution in Hidalgo. BJ $=$ Baja California, $\mathbf{C A L}=$ California, $\mathbf{T A M}=$ Tamaulipas, $\mathbf{S O N}=$ Sonora, $\mathbf{S M S}=$ Sierra Madre del Sur, $\mathbf{P Y}=$ Yucatán Peninsula, $\mathbf{C H I}=$ Chiapas, $\mathbf{D B}=$ Balsas Basin, $\mathbf{C P M}=$ Mexican Pacific Coast, $\mathbf{S M O}=$ Sierra Madre Occidental, $\mathbf{F V M}=$ Transmexican Volcanic Belt, $\mathbf{A M}=$ Mexican Plateau, $\mathbf{S M O R}=$ Sierra Madre Oriental y $\mathbf{G M}=$ Mexican Gulf. Clade A includes the 14 mexican provinces, clade B shows relationships of the provinces in Hidalgo. Black circles indicate sinapomorfies for the generalized tracks: 14=Crotalus aquilus, $\mathbf{1 8}=$ Crotalus ravus, $\mathbf{6 1}=$ Storeira hidalgoensis, $\mathbf{7 5}=$ Thamnophis sumichrasti, $\mathbf{3 1}=$ Geophis mutitorques, 32=Geophis semidoliatus, $\mathbf{4 4}=$ Micrurus tener, $\mathbf{5 5}=$ Rhadinea gaigeae, $\mathbf{2}=$ Agkistrodon taylori, $\mathbf{2 0}=$ Crotalus totonacus, $\mathbf{4 2}=$ Micrurus bernadi, 51 $=$ Pliocercus elapoides, $\mathbf{5 6}=$ Rhadinaea marcellae, $\mathbf{5 7}=$ Rhadinaea quinquelineata, $\mathbf{7 8}=$ Chersodromus rubriventris $\mathbf{8 0}=$ Geophis turbidus $\mathbf{8 1}=$ Lampropeltis ruthveni, white circles indicate homoplasies. Bootstrap values are shown below nodes.

Mexicano, y por último éstas forman el grupo hermano de la Faja Volcánica Transmexicana.

El trazo generalizado obtenido quedó integrado solo por la superposición de los trazos individuales de tres especies: A. taylori, $C$. totonacus y $P$. elapoides los cuales formaron un patrón congruente (Fig. 2). El trazo se localizó en el sureste de Nuevo León, sur de San Luis Potosí, noreste y sureste de Hidalgo hasta llegar a Puebla, e incorpora los componentes bióticos Transicional y Neotropical, en las provincias de la Sierra Madre Oriental y la región norte del Golfo de México.
Con el AE se obtuvieron dos áreas de endemismo con un valor de 2.71-2.96 y 2.843.09. Estas dos áreas de endemismo y las especies que las conforman, permitieron identificar dos trazos generalizados. El primer trazo generalizado está constituido por la superposición de tres trazos individuales correspondientes a las especies Micrurus bernadi, Rhadinaea marcellae y $R$. quinquelineata. Este trazo se localiza en el sur de San Luis Potosí, noreste y sureste del estado de Hidalgo, así como el norte del estado de Puebla; se ubica dentro del componente biótico Transicional que incluye 


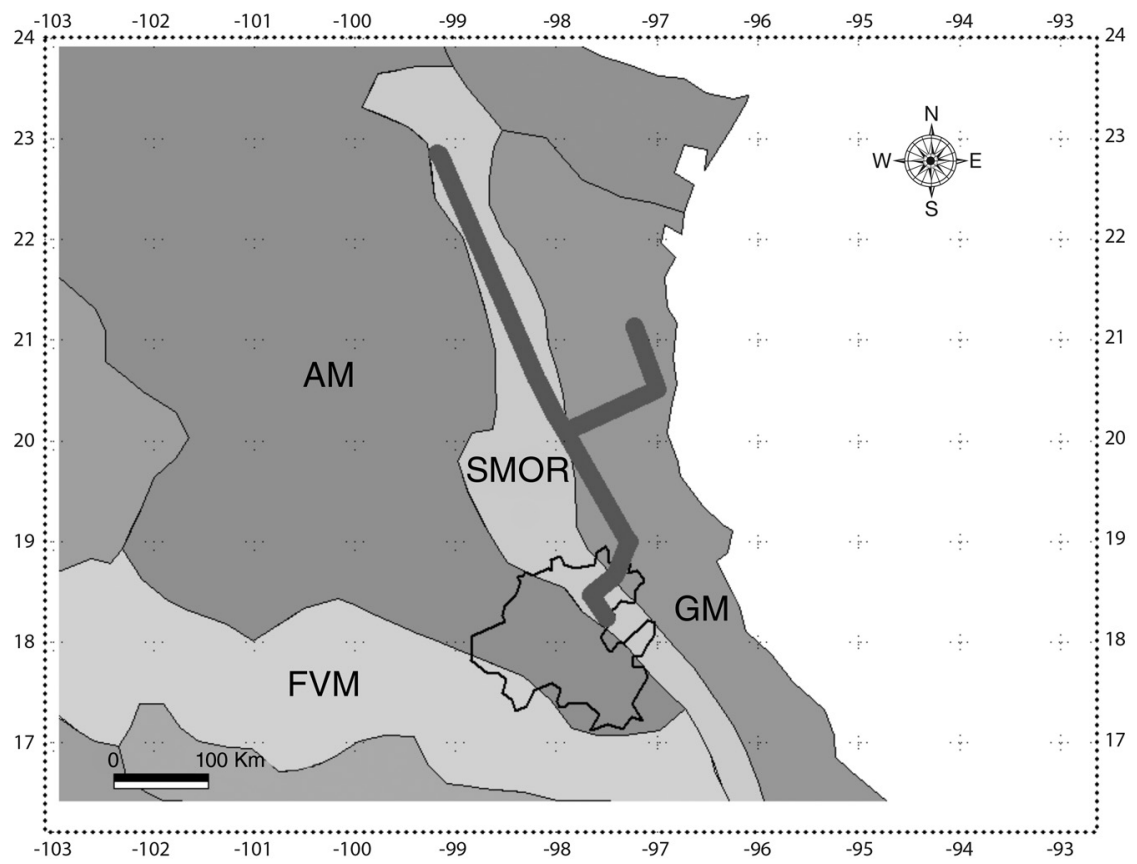

Fig. 2. Trazo generalizado obtenido como resultado del PAE, ubicado en las provincias biogeográficas de la Sierra Madre Oriental y el norte del Golfo de México. El estado de Hidalgo se muestra delineado en negro. Las abreviaturas de las provincias corresponden con la figura 1.

Fig. 2. Generalized track obtained with PAE, located in the biogeographical provinces of Sierra Madre Oriental and northern Mexican Gulf. Hidalgo State is delineated in black. Provinces abbreviations corresponds to figure 1.

la porción sur de la Sierra Madre Oriental. El segundo trazo generalizado se formó por la superposición de los trazos individuales de Geophis mutitorques y Thamnophis sumichras$t i$. Se localiza en la parte noreste de Querétaro, sur de San Luis Potosí, noreste y sureste de Hidalgo, así como el norte de Puebla hasta llegar al centro de Veracruz; se ubica geográficamente de manera similar al trazo generalizado 1, dentro del componente biótico Transicional en la porción sur de la Sierra Madre Oriental, aunque entra ligeramente al centro de la provincia del Golfo de México. Ambos trazos generalizados se encuentran muy cercanos uno de otro, por lo que al superponerse pueden considerarse como un solo patrón de distribución (Fig. 3), aunque solo se sobreponen en ciertas partes debido al tamaño de cuadrícula usada.

Al analizar los datos con un tamaño de celda mayor (1 x 1), el patrón identificado con las cuadrículas de $0.5^{\circ} \times 0.5^{\circ}$ se volvió a recuperar. El área consenso equivalente al trazo generalizado tuvo un valor de 3.06-3.97 y estuvo integrada por las mismas cinco especies que soportaron el trazo con la escala más fina. Adicionalmente, se recuperaron otras ocho áreas consenso. Sin embargo, los valores de IE de las especies que integraron dichas áreas consenso, en general, fueron menores a 0.75 , por lo tanto no se consideraron trazos generalizados.

\section{DISCUSIÓN}

Las especies que formaron los trazos generalizados en ambos métodos, pertenecen a siete géneros de cuatro familias diferentes (Elapidae, Dipsadidae, Natricidae y Viperidae) poco relacionadas filogenéticamente pero que comparten la misma distribución, esto es, que tienen concordancia geográfica debido a que respondieron de igual manera a diferentes procesos 


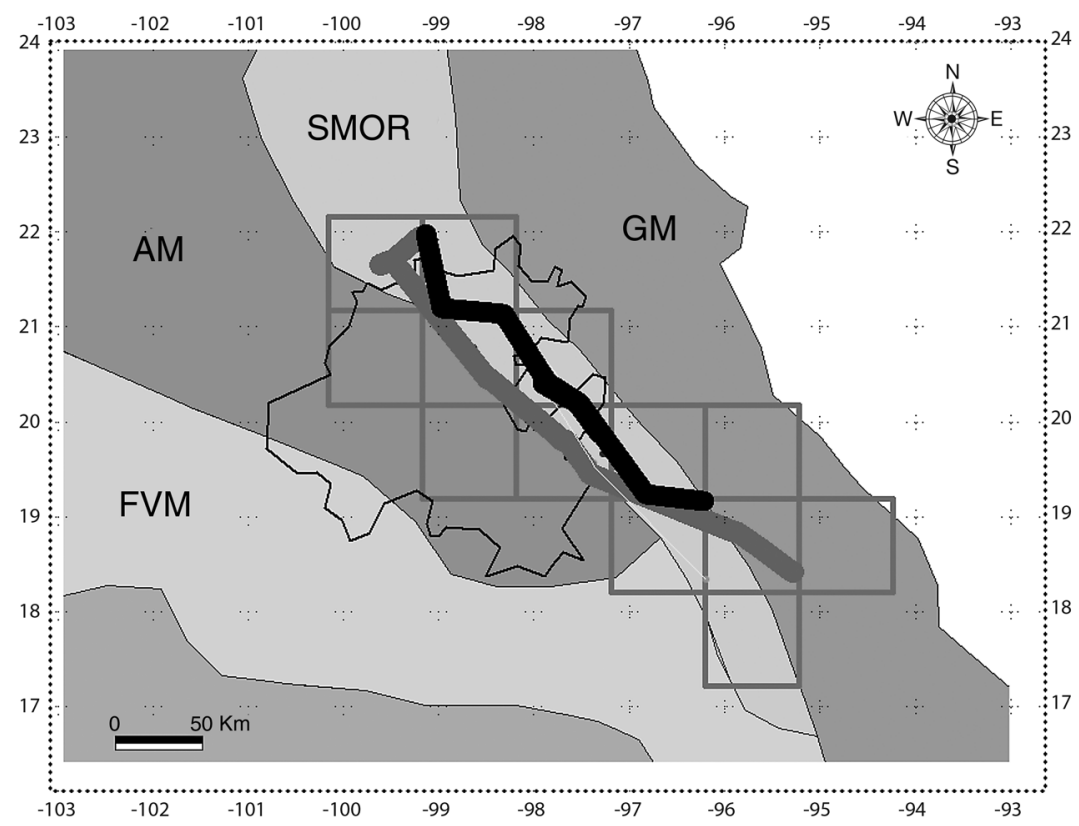

Fig. 3. Áreas de endemismo identificadas (cuadrícula) y los dos trazos generalizados obtenidos como resultado del AE, ubicados principalmente en la región sur de la Sierra Madre Oriental, en donde se muestra el mismo patrón de distribución. El estado de Hidalgo se muestra delineado en negro. Las abreviaturas de las provincias corresponden con la figura 1.

Fig. 3. Endemicity areas recovered (grid cells) and both generalized tracks obtained with the EA, mainly located in the southern region of the Sierra Madre Oriental province, showing the same distribution pattern. Hidalgo State is delineated in black. Provinces abbreviations corresponds to figure 1 .

tanto ecológicos como históricos, por lo cual muestran un patrón de distribución similar en espacio y tiempo (Fa \& Morales, 1998; Espinosa, Aguilar, \& Escalante, 2001; Zunino \& Zullini, 2003; García-Marmolejo et al., 2008). Como lo mencionan Flores-Villela y Goyenechea (2003), Halas, Zamparo y Brooks (2005) y Morrone (2005), la biota de México es muy compleja y variada puesto que en ella se superponen elementos bióticos de afinidad neártica y neotropical, lo que resulta en una interesante mezcla biótica, con un gran número de táxones endémicos. De modo similar la ofidiofauna distribuida en Hidalgo está integrada por elementos norteamericanos (p. ej. Crotalus molossus), sudamericanos (p. ej. Bothrops asper) y endémicos (p. ej. Geophis mutitorques, Rhadinaea marcellae, $R$. quinquelineata, Micrurus bernadi), lo que muestra la complejidad de la historia biogeográfica del área (Morrone, 2005; Flores-Villela \& Martínez-Salazar, 2009).
Se encontró un número distinto de trazos con los dos métodos. Con el PAE se obtuvo solo un trazo generalizado, y se usaron las provincias biogeográficas. En este análisis, las provincias del Golfo de México y la Sierra Madre Oriental resultaron las áreas más relacionadas, lo que concuerda con lo reportado por Hernández-Salinas (2009) en su análisis de los reptiles de Hidalgo. La Sierra Madre Oriental se considera la provincia biogeográfica más importante para las serpientes distribuidas en Hidalgo pues en el cladograma se sustenta por cuatro especies exclusivas. Además, en ella se registra el mayor número de ofidios con distribución restringida en dicha provincia, lo que concuerda con Canseco-Márquez, Mendoza-Quijano y Gutiérrez-Mayén (2004) y Hernández-Salinas (2009).

Las especies que integran el trazo generalizado obtenido con el PAE cuentan con una distribución más amplia en comparación 
con las especies del patrón obtenido con el $\mathrm{AE}$ y se les pueden encontrar no solo en la provincia biogeográfica de la Sierra Madre Oriental, sino también en algunas regiones de la provincia del Golfo de México y son Agkistrodon taylori, que se ha registrado en bosque tropical caducifolio y bosque tropical subpernnifolio, en altitudes que van desde 160 a $360 \mathrm{~m}$ de altitud (Campbell \& Lamar, 2004); Crotalus totonacus que se encuentra en matorrales xerófilos, pastizales, bosques de pino encino y en bosque mesófilo de montaña (Cambell \& Lamar, 2004) y Pliocercus elapoides que se encuentra en el bosque de pino y el bosque mesófilo de montaña en altitudes entre 561 y 1578 m (Ramírez Bautista et al., 2014).

Por su parte, con el AE se identificaron dos áreas de endemismo que equivalen a dos trazos generalizados. El índice de endemicidad (IE) para la primera área (2.71-2.96) es ligeramente menor que el IE para la segunda (2.84-3.09), lo cual se debe al número de especies que las integran y al valor que cada especie aporta al área de endemismo. Los dos trazos generalizados en conjunto conforman un mismo patrón de distribución en la parte sur de la Sierra Madre Oriental, lo que concuerda con el trabajo de Márquez y Morrone, (2004) quienes proponen que la SMO está dividida en dos porciones (norte y sur) a partir del análisis de la distribución de coleópteros. Al interior del estado, éste se ubica en el noreste y sureste de Hidalgo. Las especies que conforman el patrón de distribución son: Micrurus bernadi, que habita en bosques templados y en los bosques mesófilos de montaña (Campbell \& Lamar, 2004); Rhadinaea marcellae, $R$. quinquelineata, Geophis mutitorques y Thamnophis sumichrasti, que se encuentran en los bosques de pino-encino y predominantemente en el bosque mesófilo de montaña (Ramírez-Bautista et al., 2014). Este patrón coincide con la Reserva de la Biosfera "RPC Corredor Biológico de Bosque Mesófilo de Montaña propuesta por la CONANP (2010) como área para la conservación, que involucra la región montañosa de la Sierra Madre Oriental a través del estado de Hidalgo y parte de los estados de Puebla y Veracruz; lo que muestra que ésta es una región con una biota compleja, en la cual se deben aplicar políticas concretas.

Micrurus bernadi es una especie que conformó parte de los trazos generalizados en ambos métodos. Sin embargo, en el PAE se descartó, ya que aunque forma parte del trazo generalizado su distribución es muy restringida, por lo que queda anidada dentro de éste $y$, según la definición, para que un trazo individual pueda considerarse como parte de un trazo generalizado debe existir una superposición significativa de las distribuciones individuales, no solo con partes de ella (Morrone, 2015); con el AE esta especie coincide con la distribución de las otras especies que conforman los trazos generalizados debido a que al utilizar tamaños de celda pequeña se pueden identificar áreas que representan trazos generalizados restringidos (Escalante et al., 2009).

Las diferencias en el número de los trazos generalizados, así como en las especies que los sustentan, pueden deberse a que cada método parte de fundamentos distintos y se realizan de modo diferente, pero ambos permiten identificar patrones congruentes de distribución, en este caso trazos generalizados. Además, pudo influir el tamaño de las unidades usadas para realizar el análisis, ya que las provincias biogeográficas usadas para el PAE son mucho mayores que las cuadrículas de $0.5^{\circ}$ usadas para el $\mathrm{AE}$, lo que permitió recuperar un patrón de distribución más grande. Otro factor que pudo influir fue el número de registros de distribución de las serpientes, dado que de algunas se contó con pocas localidades de recolecta, por lo que para el análisis con PAE se utilizaron unidades naturales, ya que el uso de cuadrículas con pocos registros podría fallar en recuperar patrones (Morrone \& Escalante, 2002), a diferencia del AE que mediante la opción de llenado permite inferir o asumir la presencia de la especie en celdas vecinas y así eliminar este sesgo del submuestreo (Szumik et al., 2006).

Otras causas por la que se pudieron obtener resultados distintos con ambos métodos pueden ser que durante la aplicación del AE se utiliza un criterio de optimización, que evalúa qué tan endémicos son los táxones que se encuentran 
dentro de un área determinada y otorga valores de endemicidad mediante un índice (Szumik et al., 2006), mientras que el PAE solo relaciona las áreas por el número de especies compartidas (Escalante \& Morrone, 2003). Nuestros resultados sugieren que el $\mathrm{AE}$ se desempeñó mejor que el PAE al recobrar patrones que se solapan entre sí (Fig. 1 y Fig. 2; Casagranda \& Thaer, 2007; Casagranda, Taher, \& Szumik, 2012). La aplicación del AE permitió identificar dos patrones de distribución de cinco serpientes con simpatría parcial entre sí, que se distribuyen a través de la región sur de la Sierra Madre Oriental y representan parcialmente un mismo patrón de distribución. Por su parte, el PAE fue incapaz de reconocer estos trazos sobrepuestos debido a que el algoritmo de simplicidad no se propuso con ese fin (Casagranda et al. 2012). Cuando se utiliza el AE se pueden modificar algunos de los parámetros para la identificación de patrones (Szumik et al., 2006; FernándezBadillo et al., 2014), como el tamaño de la celda, el valor del índice de endemicidad con el que se seleccionan las áreas, o los valores de presencias asumidas e inferidas. Así, con tamaños pequeños de celdas es posible identificar áreas que representan trazos generalizados con menores longitudes, tal como ocurrió con los dos trazos identificados, los cuales se consideran un solo patrón de distribución si se aumenta el tamaño de la celda. Por el contrario, con el PAE solo se puede modificar el tamaño de la celda con lo que en general se obtienen cladogramas menos resueltos (Casagranda et al., 2012), o trazos generalizados más grandes, mediante un tamaño de celda más grande o con regiones geográficas naturales.

Muchos autores han publicado críticas al PAE, entre las que se enfatiza la forma en la que el método identifica a las áreas de endemismo, pues el criterio de optimización que se utiliza para seleccionar áreas es a posteriori, por lo que en este sentido se ha sido considerado como un método menos apropiado que el AE (Casagranda et al., 2012; Morrone, 2014). Otros autores (Escalante \& Morrone, 2003; Nihei, 2006; Morrone, 2009; Echeverry \& Morrone, 2010; Escalante, 2011; Crother \& Murray, 2013) por su parte lo han defendido en el sentido de que lo consideran un método eficaz para encontrar patrones y, de acuerdo con Ferrari, Barão y Simões (2013) y Morrone (2015), se considera el mejor método para identificar trazos generalizados. Morrone (2014) menciona que muchas de las críticas al PAE se deben a un mal entendimiento de sus bases metodológicas, aunque reconoce que el $\mathrm{AE}$ resulta ser más eficiente que el PAE en la identificación de las áreas de endemismo. Además, si las áreas de endemismo se consideran análogas a trazos generalizados pequeños (Escalante, 2009), cuando se utiliza el método del AE con la propuesta de Fernández-Badillo et al. (2014) para identificar trazos generalizados, el AE es capaz de recobrar patrones que el PAE no puede identificar, por ejemplo áreas sobrepuestas o anidadas, tal como los dos trazos generalizados encontrados en el presente trabajo. Debido a que el AE incluye explícitamente el componente espacial, puede detectar las congruencias distribucionales y permite identificar áreas disyuntas. Por otro lado, dado que el PAE usa el criterio de máxima simplicidad, minimiza el número de homoplasias, por lo que le resulta poco probable identificar áreas disyuntas (Casagranda et al., 2012) y podría entonces usarse el PAE-PCE (García-Barros et al., 2002).

Cuando se identifican trazos generalizados pequeños como los obtenidos con el $\mathrm{AE}$, estos pueden anidarse dentro de otros más grandes como el obtenido con el PAE. En el caso particular de las serpientes del estado de Hidalgo, la identificación de éstos trazos generalizados pequeños (con cuadrículas de $0.5^{\circ}$ o menores), pueden resultar ideales para la búsqueda de patrones biogeográficos a través de limites geopolíticos y ésta información puede favorecer la realización de propuestas de conservación a nivel municipal y estatal, puesto que este enfoque aporta información de componentes históricos y actuales de los patrones de distribución del área de estudio (Contreras-Medina, Morrone, \& Luna Vega, 2001).

En conclusión, a pesar de sus diferencias conceptuales, ambos métodos pueden usarse de manera complementaria para identificar trazos 
generalizados, dado que cada uno de ellos aporta información adicional que el otro no logra encontrar. El PAE recobra relaciones de homología biogeográfica primaria (Morrone, 2004; 2015) a escalas mayores, mientras que el AE con la propuesta de Fernández-Badillo et al. (2014) recobra patrones más finos, por ejemplo áreas sobrepuestas o anidadas. Esto puede deberse a que los eventos vicariantes que formaron la distribución de las distintas familias de serpientes de Hidalgo propician una congruencia geográfica, que se traduce en los trazos generalizados encontrados. Sin embargo, ésta congruencia geográfica no es completa dado que no todas las especies responden de la misma forma a dichos eventos ni todas se han diversificado en los mismos tiempos. Por ejemplo, se tienen registros de los géneros Crotalus y Thamnophis, durante todo el Cenozoico (Mioceno, Plioceno y Pleistoceno), mientras que el género Micrurus solo se ha registrado para el Mioceno y Pleistoceno y Rhadinaea se ha registrado solo para el Pleistoceno (Goyenechea \& Castillo-Cerón, 2002). Los trazos generalizados representan distintos procesos evolutivos (vicarianza y dispersión posterior, congruencia geográfica debida a eventos geológicos, etc; Escalante, Morrone, \& Rodríguez-Tapia, 2013).

\section{AGRADECIMIENTOS}

A Norma Manríquez Morán por la revisión crítica al manuscrito, a Renate Schulz por la revisión del abstract y a dos revisores anónimos que revisaron una versión preliminar del manuscrito. Al proyecto FOMIX-CONACyT 191908 "Diversidad biológica del estado de Hidalgo" (tercera etapa) por el apoyo financiero y logístico para la realización del trabajo.

\section{RESUMEN}

Uno de los conceptos centrales en la Panbiogeografía es el trazo generalizado, el cual representa una biota ancestral que se ha fragmentado por eventos geológicos y puede recuperarse a través de distintos métodos, que incluyen el análisis de simplicidad de endemismos (PAE) y el análisis de endemicidad (AE). El PAE es un método frecuentemente utilizado para la identificación de trazos generalizados, mientras que el $\mathrm{AE}$ fue diseñado para encontrar áreas de endemismo, pero recientemente se ha usado también para identificar trazos generalizados. En este trabajo se evaluaron ambos métodos para la identificación de los trazos generalizados a partir de los datos de 84 serpientes distribuidas en el estado de Hidalgo, México. Con el PAE se obtuvo un trazo generalizado formado por tres trazos individuales (Agkistrodon taylori, Crotalus totonacus y Pliocercus elapoides), que corresponden a sinapomorfías que apoyan al clado principal. La prueba de robustez estadística mostró un soporte del $89 \%$, mientras que con el AE se identificaron dos áreas de endemismo con valores del índice de endemicidad de 2.71-2.96 y 2.843.09 respectivamente, las cuales fueron transformadas a trazos generalizados; el primero formado por tres trazos individuales (Micrurus bernadi, Rhadinaea marcellae y $R$. quinquelineata) y el segundo formado por dos trazos individuales (Geophis mutitorques y Thamnophis sumichrasti). Estos trazos generalizados pueden considerarse un solo patrón de distribución, dada la cercanía geográfica y su topología. Al compararse los métodos se observó que ambos son útiles para la identificación de trazos generalizados, y aunque son independientes, se sugiere su uso de manera complementaria. Sin embargo, para una correcta aplicación e interpretación de los resultados, es necesario considerar las bases teóricas de cada método, así como la correcta elección del tamaño de las unidades de estudio. En el caso particular del AE si se utilizan tamaños de celda pequeños éstos pueden resultar ideales para la búsqueda de patrones biogeográficos a través de límites geopolíticos y esta información puede favorecer la realización de propuestas de conservación a nivel estatal que consideren a las especies con distribuciones restringidas, pues la pérdida de éstas representaría la extinción de linajes únicos.

Palabras clave: México, panbiogeografía, componentes bióticos, análisis de simplicidad de endemismos, análisis de endemicidad.

\section{REFERENCIAS}

Aagesen, L., Szumik, C., \& Goloboff, P. (2013). Consensus in the search for areas of endemism. Journal of Biogeography, 40(11), doi:10.1111/jbi.12172

Almazán-Núñez, R., López-De Aquino, S., Ríos-Muñoz, C., \& Navarro-Sigüenza, A. (2013). Áreas potenciales de riqueza, endemismo y conservación de las aves del estado de Querétaro, México. Interciencia, $38,26-34$.

Canseco-Márquez, L., Mendoza-Quijano, F., \& GutiérrezMayén, G. (2004). Análisis de la distribución de la herpetofauna. In I. Luna-Vega, J. J. Morrone, \& D. Espinosa (Eds.), Biodiversidad de la Sierra Madre Oriental (pp. 417-437). México, D. F.: 
Las Prensas de Ciencias, Universidad Nacional Autónoma de México.

Calvanti, M. J. (2009). Croizat: A software package for quantitative analysis in panbiogeography. Biogeografica, 4, 4-6.

Campbell, J. A., \& Lamar, W. W. (2004). The venomous reptiles of the western hemisphere. Ithaca, New York: Comstock.

Casagranda, M. D., \& Taher, L. (2007). Elementos bióticos, endemismo y parsimonia: estudio de casos de distribuciones hipotéticas. Darwiniana, 45, 37-39.

Casagranda, M. D., Taher, L., \& Szumik, C. (2012). Endemicity analysis, parsimony and biotic elements: a formal comparison using hypothetical distributions. Cladistics, 28, 645-654.

Contreras-Medina, R., Morrone, J. J., \& Luna Vega, I. (2001). Biogeographic methods identify gymnosperm biodiversity hotspots. Naturwissenschaften, $88,427-430$

Comisión Nacional para el conocimiento y uso de la Biodiversidad (CONABIO). (2004). Fichas de Especies. Estatus y conservación de algunos anfibios y reptiles de México. México, D.F.: Facultad de Estudios Superiores Iztacala, Unidad de Biología, Tecnología y Prototipos (UBIPRO), Universidad Nacional Autónoma de México. Bases de datos SNIBCONABIO. Proyecto W043.

Comisión Nacional de Areas Naturales Protegidas (CONANP). (2010). Estudio Previo Justificativo para el establecimiento del Área Natural Protegida Reserva de la Biosfera "Corredor Biológico del Bosque Mesófilo de Montaña en Hidalgo, Puebla y Veracruz" México: Comisión Nacional de Áreas Naturales Protegidas.

Cué-Bär, E. M., Villaseñor, J. L., Morrone, J. J., \& IbarraManríquez, G. (2006). Identifying priority areas for conservation in Mexican tropical deciduous forest on tree species. Interciencia, 31, 712-719.

Craw, R. C. (1988). Continuing the synthesis between panbiogeography, phylogenetic systematics and geology as illustrated by empirical studies on the biogeography of New Zealand and the Chatham Islands. Systematic Zoology, 37, 291-310.

Craw, R. C., Grehan, J. R., \& Heads, M. J. (1999). Panbiogeography; tracking the history of life. New York: Oxford University Press.

Crisci, J. V., Katinas, L., \& Posadas, P. (2000). Introducción a la teoría y práctica de la biogeografia histórica. Buenos Aires: Sociedad Argentina de Botánica.

Croizat, L. (1958). Panbiogeography. Vols. 1 y 2. Caracas: Publicado por el autor.
Croizat, L. (1964). Space, time, form: The biological synthesis. Caracas: Publicado por el autor.

Crother, B. I., \& Murray, C. M. (2013). Parsimony analysis of endemism under the 'areas of endemism as individuals' thesis. Cladistics, 29,571-573.

Darwin, C. R. (1859). The origin of species by means of natural selection or the preservation of favoured races in the struggle for life. London: John Murray.

Delgado, L., \& Márquez, J. (2006). Estado del conocimiento y conservación de los coleópteros Scarabaenidae (Insecta) del estado de Hidalgo. Acta Zoológica Mexicana (Nueva serie), 22, 57-108.

Echeverría-Londoño, S., \& Miranda-Esquivel, D. R. (2011). MartiTracks: A geometrical approach for identifying geographical patterns of distribution. $\mathrm{PloS}$ One 6: e18460. doi: 10.1371/journal.pone.0018460.

Echeverry, A., \& Morrone, J. J. (2010). Parsimony analysis of endemicity as a panbiogeographical tool: an analysis of Caribbean plant taxa. Biological Journal of the Linnean Society, 101, 961-976.

Escalante, T. (2009). Un ensayo sobre la regionalización biogeográfica. Revista Mexicana de Biodiversidad, 80, 817-833.

Escalante, T. (2011). De cómo el análisis de parsimonia de endemismos (PAE) tampoco explica la selección natural. Revista Mexicana de Biodiversidad, 82, 1057-1059.

Escalante, T. \& Morrone, J. J. (2003). ¿Para qué sirve el Análisis de Parsimonia de Endemismos? In J. J. Morrone \& J. Llorente (Eds.), Una perspectiva latinoamericana de la biogeografia (pp. 167-172). México, D.F.: Las Prensas de Ciencias, Facultad de Ciencias, Universidad Nacional Autónoma de México.

Escalante, T., Szumik, C., \& Morrone, J. J. (2007). Áreas de endemismo de los mamíferos de México: reanálisis y comparación con estudios previos. Darwiniana, $45,41-43$.

Escalante, T., Szumik, C., \& Morrone, J. J. (2009). Areas of endemism of Mexican mammals: reanalysis applying the optimality criterion. Biological Journal of the Linnean Society, 98, 468-478.

Escalante, T., Morrone, J. J., \& Rodríguez-Tapia, G. (2013). Biogeographic regions of North American mammals based on endemism. Biological Journal of the Linnean Society, 110, 485-499.

Espinosa, D., Aguilar, C., \& Escalante, T. (2001). Endemismo, áreas de endemismo y regionalización biogeográfica. In J. Llorente \& J. J. Morrone (Eds.), Introducción a la biogeografía en Latinoamérica: teorías, conceptos, métodos y aplicaciones (pp. 31-37). México, D. F.: Las Prensas de Ciencias, 
Facultad de Ciencias, Universidad Nacional Autónoma de México, México.

ESRI. (1998). ArcView GIS 3.2. Environmental Systems Research Institute. Redlands, California.

Fa, J. E., \& Morales, L. M. (1998). Patrones de diversidad de mamíferos de México. En T. P. Ramamoorthy, R. Bye, A. Lot, \& J. E. Fa (Eds.), Diversidad biológica de México: orígenes y distribución (pp. 22-65). México, D. F.: Instituto de Biología, Universidad Nacional Autónoma de México.

Ferrari, A., Barão, K. R., \& Simões, F. L. (2013). Quantitative panbiogeography: was the congruence problem solved? Systematics and Biodiversity, 11, 285-302.

Fernández-Badillo, L. (2013). Patrones de distribución de las lagartijas y las serpientes en los desiertos mexicanos (Tesis de Maestría). Universidad Autónoma del Estado de Hidalgo, Pachuca, Hidalgo, México.

Fernández-Badillo, L., Goyenechea, I., \& Escalante, T. (2014). ¿Puede el análisis de endemicidad usarse para la identificación de trazos generalizados? Biogeografia, 7, 15-26.

Flores-Villela, O., \& Goyenechea, I. (2003). Patrones de distribución de anfibios y reptiles en México. In J. J. Morrone \& J. Llorente (Eds.), Una perspectiva latinoamericana de la biogeografia (pp. 989-296). México, D.F.: Las Prensas de Ciencias, Facultad de Ciencias, Universidad Nacional Autónoma de México.

Flores-Villela, O., \& Martínez-Salazar, E. A. (2009). Historical explanation of the origin of the herpetofauna of Mexico. Revista Mexicana de Biodiversidad, $80,817-833$.

García-Barros, E., Gurrea, P., Luciañez, M., Cano, J., Munguira, M., Moreno, J., Sainz, H., Sanz, M., \& Simón, J. C. (2002). Parsimony analysis of endemicity and its application to animal and plant geographical distributions in the Ibero-Balearic region (western Mediterranean). Journal of Biogeography, 29, 109-124.

García-Marmolejo, G., Escalante, T., \& Morrone, J. J. (2008). Establecimiento de prioridades para la conservación de los mamíferos terrestres neotropicales de México. Mastozoología Mexicana, 15, 41-65.

Global Biodiversity Information Facility (GBIF). (2014) Recuperado de www.gbif.org

Goloboff, P. A. (2014). NDM/VNDM Ver 3.0 Programas para la identificación de áreas de endemismo. Recuperado de http://www.lillo.org.ar/phylogeny/endemism/

Goloboff, P. A., Farris, J. S., \& Nixon, K. C. (2008). TNT, a free program for phylogenetic analysis. Cladistics, 24, 1-13.
Goyenechea, I., \& Castillo-Cerón, J. M. (2002). Historia geológica de las serpientes. Boletín de la Sociedad Herpetológica Mexicana, 10, 35-42.

Halas, D., Zamparo, D., \& Brooks, D. R. (2005). A protocol for studying biotic diversification by taxon pulses. Journal of Biogeography, 32, 249-260.

Hernández-Salinas, U. (2009). Estudio herpetofaunístico del estado de Hidalgo, México (Tesis de Maestría). Universidad Autónoma del Estado de Hidalgo. Pachuca, Hidalgo.

Instituto nacional de estadística y geografía (INEGI). (2009). El INEGI en tu entidad. Hidalgo-Geografía. Recuperado de http://www.inegi.org.mx/inegi/ default.aspx?s=geo\&e $=22$

International Union for Conservation of Nature (IUCN). (2014). The 2014 IUCN red list of threatened species. Version 2014. The world conservation. Recuperado de http://www.iucnredlist.org

Liria, J. (2008). Sistemas de información geográfica y análisis espaciales: un método combinado para realizar estudios panbiogeográficos. Revista Mexicana de Biodiversidad, 79, 281-284.

Luna-Vega, I., Alcántara, O., Morrone, J. J., \& Espinosa, D. (2000). Track analysis conservation priorities in the cloud forest of Hidalgo, Mexico. Diversity and Distributions, 6, 137-143.

Márquez, J., \& Morrone, J. J. (2004). Relaciones biogeográficas basadas en la distribución de Coleoptera (Insecta). In I. Luna-Vega, J. J. Morrone y D. Espinosa (Eds.), Biodiversidad de la Sierra Madre Oriental (pp. 375-392). México, D. F.: Las Prensas de Ciencias, Universidad Nacional Autónoma de México.

Morrone, J. J. (1994). On the identification of areas of endemism. Systematic Biology, 43, 438-441.

Morrone, J. J. (2000). Entre el escarnio y el encomio: León Croizat y la panbiogeografía. Interciencia, 25, 41-47.

Morrone, J. J. (2001). Biogeografía de América Latina y el Caribe. Manuales y Tesis 3. Zaragoza: Sociedad Entomológica Aragonesa.

Morrone, J. J., \& Escalante, T. (2002). Parsimony analysis of endemicity (PAE) of Mexican terrestrial mammals at different area units: when size matters. Journal of Biogeography, 29, 1095-1104.

Morrone, J. J. (2003). El lenguaje de la cladística. México, D. F.: Las Prensas de Ciencias, Facultad de Ciencias. Universidad Nacional Autónoma de México.

Morrone, J. J. (2004). Panbiogeografía, componentes bióticos y zonas de transición. Revista Brasileira de Entomología, 48, 149-162. 
Morrone, J. J. (2005). Hacia una síntesis biogeográfica de México. Revista Mexicana de Biodiversidad, 76, 207-252.

Morrone, J. J. (2009). Evolutionary biogeography: an integrative approach with case studies. New York: Columbia University Press.

Morrone, J. J., \& Escalante, T. (2009). Panbiogeografía y biogeografía cladística: Enfoques complementarios para entender la evolución biótica. In J. J. Morrone, \& P. Magaña (Eds.), Evolución biológica: Una visión actualizada desde la revista Ciencias (pp. 385-386). México, D. F.: Universidad Nacional Autónoma de México.

Morrone, J. J. (2014). Parsimony analysis of endemicity (PAE) revisited. Journal of Biogeography, $41,842-854$.

Morrone, J. J. (2015). Track analysis beyond Panbiogeography. Journal of Biogeography, 42, 413-425.

Nihei, S. S. (2006). Misconceptions about parsimony analysis of endemicity. Journal of Biogeography, 33, 2099-2106.

Page, R. D. M. (1987). Graphs and generalized tracks: quantifying Croizat's panbiogeography. Systematic Zoology, 36, 1-17.

Ramírez-Bautista, A., Hernández-Salinas, U., GarcíaChávez, U. O., Leyte-Manrique, A., \& CansecoMárquez, L. (2009). Herpetofauna del Valle de México: Diversidad y conservación. México, D. F.: Comisión Nacional para el Conocimiento y Uso de la Biodiversidad y Universidad Autónoma del Estado de Hidalgo.

Ramírez-Bautista, A., Hernández-Salinas, U., Cruz-Elizalde, R., Berriozabal-Islas, C. S., Lara-Tufiño, D., Goyenechea, I., \& Castillo-Cerón, J. (2014). Los anfibios y reptiles del estado de Hidalgo, México: diversidad, biogeografia y conservación. México, D.F.: Sociedad Herpetológica Mexicana, A. C.

Rojas-Parra, C. A., Poveda-Matallana, I. C., Prieto-Cruz, A., Rudas-Lleras, A., \& Luis-Martínez, M. A. (2003). El tamaño de la celda en los análisis de patrones espaciales de la biodiversidad utilizando sistemas de información geográfica: ¿Un Problema de escalas? In J. J. Morrone \& J. Llorente (Eds.), Una perspectiva latinoamericana de la biogeografia (pp. 123-140). México, D.F.: Las Prensas de Ciencias, Facultad de Ciencias, Universidad Nacional Autónoma de México.

Rojas-Parra, C. A. (2007). Una herramienta automatizada para realizar análisis panbiogeográficos. Biogeografia, 1, 31-33.

Szumik, C. A., Cuezzo, F., Goloboff, P. A., \& Chalup, A. E. (2002). An optimality criterion to determine areas of endemism. Systematic Biology, 51, 806-316.

Szumik, C., \& Goloboff, P. A. (2004). Areas of endemism. An improved optimally criterion. Systematic Biology, 53, 968-977.

Szumik, C., Casagranda, D., \& Roig-Juñent, S. (2006). Manual de NDM/VNDM: Programas para la identificación de áreas de endemismo. Instituto Argentino de Estudios Filogenéticos, 5, 1-26.

Uetz, P., \& Hošek, J. (2014). The reptile data base. Recuperado de http://www.reptile-database

VertNet (2014). Proyecto Vert Net, National Science Foundation. Recuperado de http://www.vertnet.org/

Wallace, A. R. (1876). The geographical distribution of animals, with a study of the relations of living and extinct faunas as elucidating the past changes of the Earth's surface. Vol. I. London: Macmillan and Company.

Wilson, L. D., Mata-Silva, V., \& Johnson, J. D. (2013). A conservation reassessment of the reptiles of Mexico based on the EVS measure. Amphibian and Reptile Conservation, 7, 1-47.

ZenScientist. (2014). Catalogue of American Amphibians and Reptiles. Recuperado de http:// www.zenscientist.com/index.php/filedrawer/ Open-Access-Journals/caar/

Zunino, M., \& Zullini, A. (2003). La dimensión espacial de la evolución. México, D. F.: Fondo de Cultura Económica. 